\title{
Nondiabetic Lumbosacral Radiculoplexus Neuropathy with Abnormal Magnetic Resonance Neurography Findings
}

\author{
Sung-Eun Lee, Yong-Woo Lee, In-Ho Yoon, Chang-Min Lee \\ Department of Neurology, Dankook University Hospital, Cheonan, Korea
}

Nondiabetic lumbosacral radiculoplexus neuropathy is a rare (sub) acute, motor disorder that mainly occurs in the lower limb, affecting multiple levels of the nerve roots, lumbosacral plexus, and distal nerves. A 48-year-old woman presented with an acute onset sharp and burning pain in the left thigh and groin, followed by progressive weakness of muscles innervated by the left femoral nerve. Magnetic resonance neurography revealed contrast enhancement and swelling of the left $\mathrm{L}_{3}$, and L4 roots and the left femoral nerve. Good response to steroids was observed.

\section{J Neurosonol Neuroimag 2019;11(1):100-103}

Key Words: Nondiabetic lumbosacral radiculoplexus neuropathy; Magnetic resonance neurography; Steroid
Received: December 20, 2018

Revised: February 8, 2019

Accepted: February 25, 2019

Address for correspondence: Chang-Min Lee

Department of Neurology, Dankook University Hospital, 119 Dandae-ro, Dongnam-gu, Cheonan 31116, Korea

Tel: +82-41-550-3990

Fax: $+82-41-550-7158$

E-mail:nrdoc@dku.edu
Non-diabetic lumbosacral radiculoplexus neuropathy (LRPN) is an uncommon disorder. It is also called idiopathic lumbosacral plexopathy or lumbosacral plexitis. Its core features are acute-to-subacute, severe, asymmetrical leg pain, followed by asymmetrical multifocal weakness and atrophy in the subsequent weeks or months. ${ }^{1}$ Compared with similar disorders, LRPN has received little attention since its first recognition in 1981. It was reported to be monophasic but with prolonged morbidity because of pain and weakness. ${ }^{2,3}$

LRPN can be diagnosed on the basis of clinical features and electrophysiological studies in the absence of other secondary causes. Magnetic resonance (MR) neurography is useful for the diagnosis of acute LRPN. To our knowledge, no case of acute LRPN with abnormal MR neurography findings has been reported in Korea.

\section{CASE REPORT}

A 48-year-old woman visited Dankook University Hospital with left lower limb pain and weakness that had persisted since 3 days prior. At first, she felt a sudden dullness in her left knee. After 1 hour, a sharp pain developed from the knee to the inside of the thigh and pelvis. After another hour, she could no longer stand owing to progressive weakness. The pain improved after intravenous injection of pain medication in the emergency department of another hospital, but the weakness and paresthesia with burning sensation did not subside. She had no history of trauma, operation, injection, diabetes, arthritis, vaccination, weight loss, or fever. She had no specific family history. She was a farmer without a smoking or drinking history.

On neurological examination, the cranial nerve was normal. In the left lower extremity, the hip joint flexion, hip joint adduction, hip joint abduction, and knee extension muscle strengths were classified as Medical Research Council (MRC) grades 2, 3, 4, and 3, respectively. She had mild loss of pain and vibration sensations in the left antero-medial thigh and medial shin. The muscle stretch reflexes were normal except for the absence of left knee jerk. Plantar responses were flexor.

The results of her laboratory tests, including complete blood counts, blood chemistry, erythrocyte sedimentation rate, and C-reactive protein level, were normal. Findings from chest radiography, electrocardiography, and urinalysis were nonspecific. Antinuclear 
antibody, rheumatoid factor, anti-ds-DNA antibody, anti-neutrophil cytoplasmic antibody and other autoimmune antibodies were all negative. Paraneoplastic antibodies and cancer markers were also negative. Immunoglobulin (Ig) G and IgM antibodies against ganglioside GM1, GDıb, and GQ1b were negative. The level of IgM antibody against myelin-associated glycoprotein was normal. Electrophoresis revealed normal serum protein levels. Cerebrospinal fluid (CSF) analysis revealed no specific findings other than a slight increase in protein level to $48.1 \mathrm{mg} / \mathrm{dL}$. The results of serological tests for hepatitis B and C and human immunodeficiency viruses and the syphilis test were negative. Gastrofiberscopy and colonoscopy findings were normal. Whole-body positron emission tomography-computed tomography (PET-CT) did not detect any abnormality. On admission, brain magnetic resonance imaging (MRI) and lumbosacral spine MRI revealed no abnormal findings.

Nerve conduction study (NCS) on the second day of admission showed no abnormal findings except increased latencies of the left posterior tibial and peroneal nerves. Electromyography (EMG) of the left vastus lateralis, adductors, tibialis anterior, gastrocnemius, and paraspinous muscles revealed no abnormalities. However, post-gadolinium fat-suppressed T1-weighted MRI of the lumbosacral plexus revealed enhancements of the left $\mathrm{L}_{3}$ and $\mathrm{L}_{4}$ roots and swelling of the left $\mathrm{L}_{4}$ root. The left femoral nerve was also thickened, with mild contrast enhancement (Fig. 1).

The patient was diagnosed as having LRPN on the basis of the clinical and MRI findings. She was administered an intravenous injection of methylprednisolone ( $1 \mathrm{~g} /$ day) for 5 days and oral prednisolone (6o mg/day). Approximately 1 week after dosing, the left lower extremity pain began to improve, and the numeric rating scale (NRS) score decreased 7 on admission to 5 and then 2 after 1 month. No significant changes were found in the strength of the other muscles. The hip joint adduction strength was improved to MRC grade
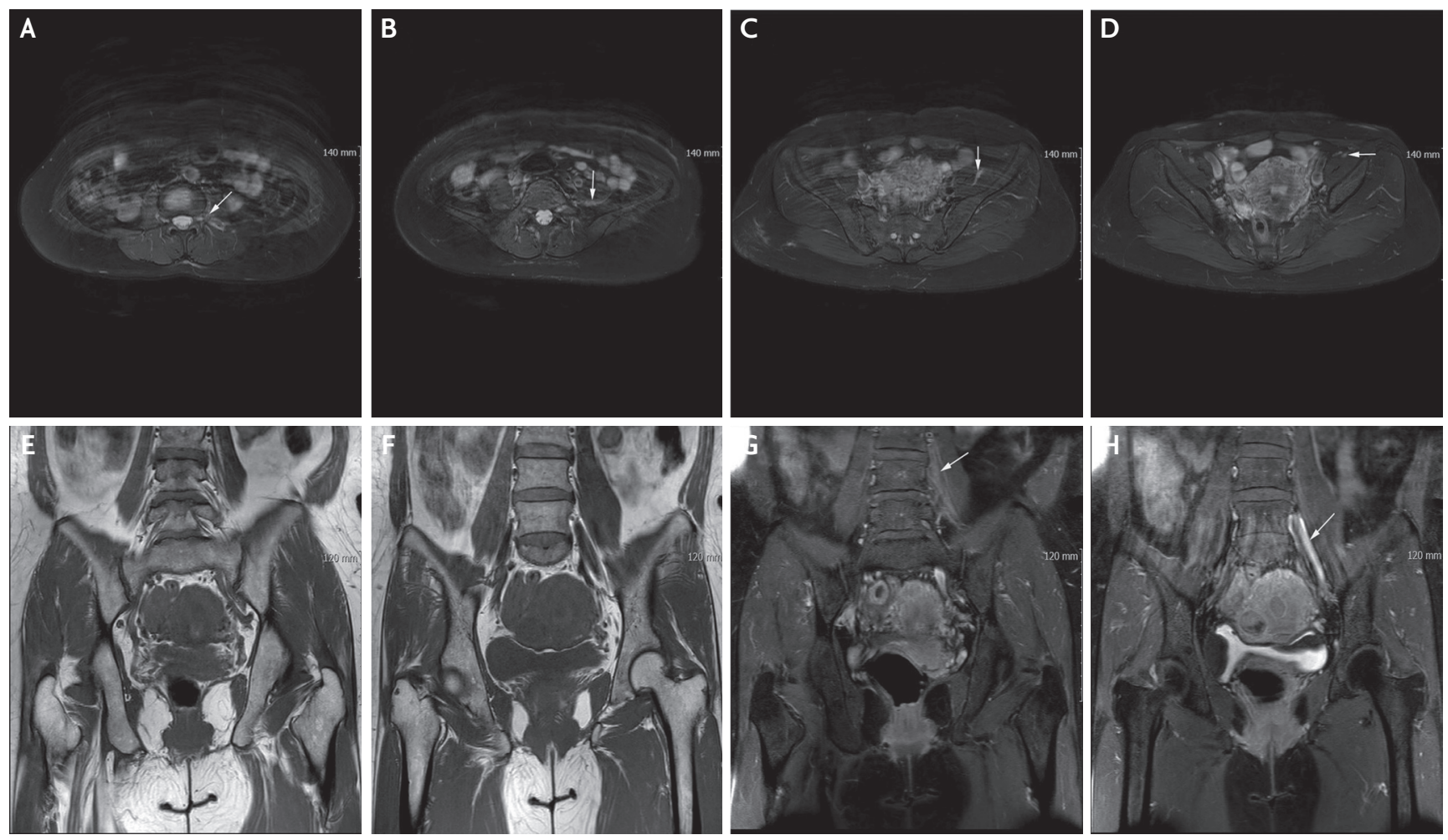

FIG. 1. Lumbosacral plexus magnetic resonance axial post-gadolinium fat-suppressed (A-D), coronal non-enhanced (E, F), and coronal post-gadolinium fat-suppressed T1-weighted images $(G, H)$. Mild enhancement of the left $L_{3}$ nerve root (arrow) is shown by the post-gadolinium axial (A) and coronal $(G)$ sections in comparison with the non-enhanced coronal image (E). Moderate enhancement and swelling of the left $L 4$ nerve root (arrow) are also shown by the post-gadolinium axial $(B)$ and coronal images $(\mathrm{H})$ in comparison with non-enhanced coronal image $(\mathrm{F})$. The left femoral nerve (arrow) is thickened with mild contrast enhancement (C, D). 
4 after 1 month. In the follow-up NCS, the amplitude of the femoral nerve compound motor action potential was 1 and $3 \mathrm{mV}$ on the left and right sides, respectively. The amplitude of the left saphenous nerve action potential was $2.5 \mu \mathrm{V}$ on the left side, which was lower than the $7.1 \mu \mathrm{V}$ on the right side. EMG revealed denervation findings from the left iliacus, vastus lateralis, and $\mathrm{L}_{3}$ and L4 paraspinal muscles, which suggested left $\mathrm{L}_{3}$ and L4 polyradiculopathy with femoral neuropathy. Over 4 months, the steroid dosage was slowly reduced, and oral prednisolone administration was maintained at 7.5 $\mathrm{mg} /$ day.

At 6 months after symptom onset, the paresthesia and pain remained in NRS 2. However, the hip joint flexion strength was MRC grade 3, hip joint adduction and knee extension strengths were MRC grade 4, and hip joint abduction strength was MRC grade 5. On follow-up lumbosacral plexus MRI, the enhancement and edema of the left $\mathrm{L}_{3}$ and $\mathrm{L}_{4}$ nerve roots and femoral nerve almost completely disappeared (Fig. 2).

\section{DISCUSSION}

The exact incidence of LRPN is unknown, but its prevalence is low. Men and women are equally affected. The incidence of diabetic LRPN (DLRPN) is estimated to account for $<1 \%$ of all diabetes cases. LRPN is predominantly a disorder among the elderly, with a median age of almost 70 years, but it can also occur at any age. Evidence on its racial or geographic predilection is currently lacking. ${ }^{1,3}$
The most common clinical symptoms of LRPN are unilateral lower limb pain, weakness, atrophy, and abnormal sensation, which occur suddenly over a period of days to weeks or months. LRPN usually begins locally in the buttock and thigh rather than in the leg or foot, and the affected area gradually spreads from one side to the other. Pain is the most severe symptom initially, but weakness becomes the more disabling symptom later. Other associated symptoms include weight loss and autonomic dysfunction. The typical autonomic dysfunctions are orthostatic hypotension, urinary dysfunction, change in sexual function, diarrhea, constipation, or change in sweating. ${ }^{3,4}$

Most patients with LRPN have normal erythrocyte sedimentation rate, rheumatoid factor level, and antinuclear antibody levels. The CSF protein concentration is significantly elevated, although the number of cells in CSF is normal., ${ }^{3,4}$ In previously reported cases, lumbosacral spine MRI or CT myelography failed to show structural lesions that could explain the neurological deficits. ${ }^{3}$ However, recently, increased signal intensity or enhancement of the nerve roots on lumbosacral plexus MRI has been reported., ${ }^{5,6}$ In addition, like the present case, cases in the acute stage are rarely diagnosed and treated with MR neurography. ${ }^{5}$

The electrophysiological abnormalities of LRPN tend to be much more widespread than the clinical deficits. These abnormalities are more suggestive of axonal degeneration than segmental demyelination. ${ }^{1,4}$ LRPN in the acute phase is difficult to diagnose with electrophysiological study because electrophysiological abnormalities appear after a certain period from nerve injury.
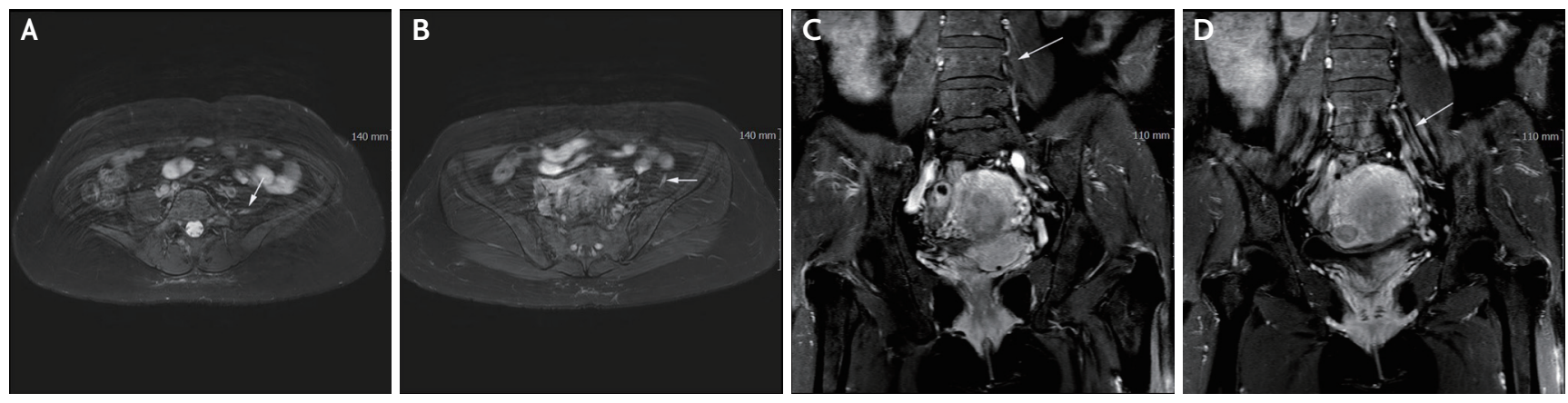

Fig. 2. Follow-up lumbosacral plexus magnetic resonance axial post-gadolinium fat-suppressed (A, B) and coronal post-gadolinium fat-suppressed T1-weighted images (C, D) 6 months later. The post-gadolinium axial (A) and coronal images (D) demonstrate decreased enhancement and swelling of the left $L_{4}$ nerve root (arrow). The post-gadolinium coronal image (C) demonstrates the disappearance of the left $L_{3}$ nerve root enhancement (arrow). No thickening of the left femoral nerve (arrow) can be observed on the post-gadolinium axial image (B). 
Moreover, our case had no abnormality in the electrophysiological study performed in the acute phase. A month later, LRPN-compatible findings were observed on follow-up electrophysiological study. Therefore, clinical features and MRI findings are more useful for the diagnosis of acute LRPN.

The etiology of LRPN is uncertain, but it is presumed to be an immune-mediated disorder. Recently, biopsy findings of the distal cutaneous nerve in patients with LRPN have shown typical findings of ischemic injury and microvasculitis which were similar to those in patients with DLRPN; this suggests that inflammation is a common pathological mechanism in the two disorders. ${ }^{7}$ In addition, immunohistochemical staining of LRPN and DLRPN nerves showed upregulation of immunological mediators such as intercellular adhesion molecule 1 (ICAM-1), tumor necrosis factor $\alpha$ (TNF- $\alpha$ ), and nuclear factor $\kappa \mathrm{B}(\mathrm{NF}-\mathrm{\kappa B}){ }^{8}$

The differential diagnoses of LRPN are cancer, benign tumor, amyloidosis, infection, trauma, radiation toxicity, hematoma, vascular lesions, and inflammatory lesions (DLRPN, postsurgical inflammatory neuropathy, and sarcoidosis). ${ }^{9}$

Treatment of LRPN is largely divided into immunotherapy and symptomatic therapy. The effectiveness of immunotherapy currently lacks evidence from a randomized controlled trial. ${ }^{1}$ Bradley et al.reported that four of six patients improved with prednisone therapy. ${ }^{4}$ Verma et al. reported that two patients responded to high-dose intravenous immunoglobulin (IVIg) therapy. ${ }^{4}$ Triggs et al. reported that five patients also showed improvement with IVIg therapy. ${ }^{4}$ Symptomatic treatment is particularly important for pain. Physical therapy and bracing should be considered.

The prognosis of LRPN depends on the extent and severity of involvement. All patients recover to some degree but usually not completely. ${ }^{10}$ Dyck et al. ${ }^{3}$ reported that 10 of 57 patients with LRPN initially underwent lumbar laminectomy for presumed lumbosacral radiculopathy from a herniated disk, but the symptoms worsened. In our case, the clinical course was relatively good, the pain was reduced rapidly, and there was only one side involvement without recurrence. Treatment was started quickly owing to the rapid diagnosis in the acute phase.

In conclusion, prompt and accurate diagnosis using
MR neurography in patients suspected of having acute LRPN can help avoid unnecessary surgery and allow appropriate treatment to be started quickly. Patients must be convinced that the disease is monophasic, with a long duration and usually incomplete recovery. Immunotherapy should be considered if symptoms are severe or progressive.

\section{Conflicts of Interest}

No potential conflicts of interest relevant to this article was reported.

\section{REFERENCES}

1. van Eijk J, Chan YC, Russell JW. Immunotherapy for idiopathic lumbosacral plexopathy. Cochrane Database Syst Rev. 2013;31:CDoo9722.

2. Sander JE, Sharp FR. Lumbosacral plexus neuritis. Neurology. 1981;31:470-473.

3. Dyck PJ, Norell JE, Dyck PJ. Non-diabetic lumbosacral radiculoplexus neuropathy: natural history, outcome and comparison with the diabetic variety. Brain. 2001;124:11971207.

4. Dyck PJ, Windebank AJ. Diabetic and nondiabetic lumbosacral radiculoplexus neuropathies: new insights into pathophysiology and treatment. Muscle Nerve. 2002;25:477-491.

5. Imam Y, Deleu D, Salem K. Idiopathic lumbosacral plexitis. Qatar Med J. 2013;2012:85-87.

6. Filosto M, Pari E, Cotelli M, Todeschini A, Vielmi V, Rinaldi $\mathrm{F}$, et al. MR neurography in diagnosing nondiabetic lumbosacral radiculoplexus neuropathy. J Neuroimaging. 2013;23:543-544.

7. Dyck PJ, Engelstad J, Norell J, Dyck PJ. Microvasculitis in non-diabetic lumbosacral radiculoplexus neuropathy (LSRPN): similarity to the diabetic variety (DLSRPN). J Neuropathol Exp Neurol. 2000;59:525-538.

8. Kawamura N, Dyck PJ, Schmeichel AM, Engelstad JK, Low PA, Dyck PJ. Inflammatory mediators in diabetic and non-diabetic lumbosacral radiculoplexus neuropathy. Acta Neuropathol. 2008;115:231-239.

9. Dyck PJ, Thaisetthawatkul P. Lumbosacral plexopathy. Continuum (Minneap Minn). 2014;20:1343-1358.

10. Dyck PJ, Norell JE, Dyck PJ. Methylprednisolone may improve lumbosacral radiculoplexus neuropathy. Can J Neurol Sci. 2001;28:224-227. 